\title{
Adsorption Thermodynamics of Short-chain Peptides on Charged and Uncharged Nanothin Polymer Films
}

Nripen Singh, Scott M. Husson*

Department of Chemical and Biomolecular Engineering, Clemson University, Clemson,

SC 29634-0909

AUTHOR EMAIL ADDRESS: shusson@clemson.edu

\section{RECEIVED DATE}

* To whom correspondence should be addressed. Tel.: +1 (864) 656-4502. Fax: +1 (864) 656-0784. E-mail: shusson@clemson.edu.

Supporting Information. Chemical structures are given for all of the peptides studied, followed by surface plasmon resonance (SPR) response curves at $25{ }^{\circ} \mathrm{C}$ and $\mathrm{pH} 7$ for all these peptides on nanothin films of poly(2-vinylpyridine) (PVP), poly(styrene) (PS), and poly(1-benzyl-2-pyridinium bromide) (BzPVP). 
Scheme 1. Structure of leucine-enkephalin (YGGFL)

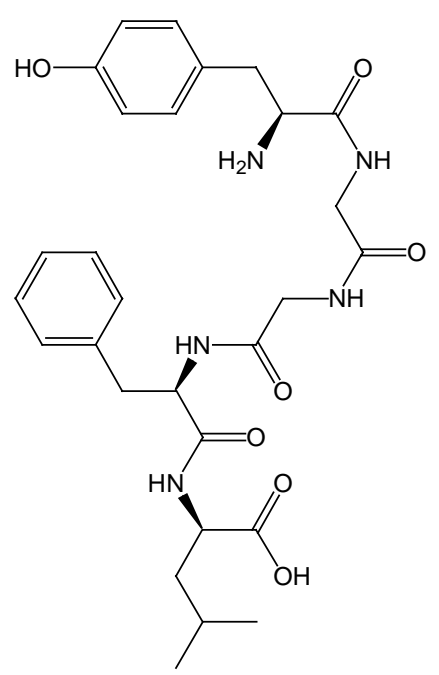


Scheme 2. Structures of (a) Tyr-Tyr (YY), (b) Tyr-Tyr-Tyr (YYY), (c) Tyr-Tyr-Tyr-TyrTyr-Tyr (YYYYYY).

(a)

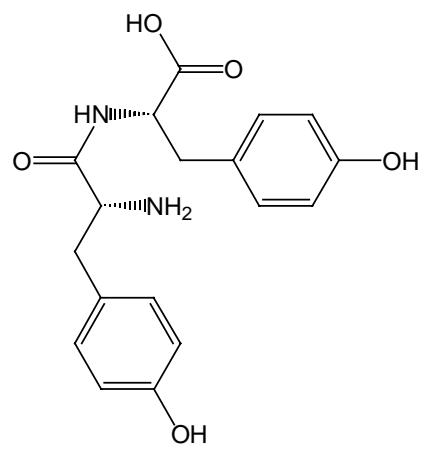

(b)

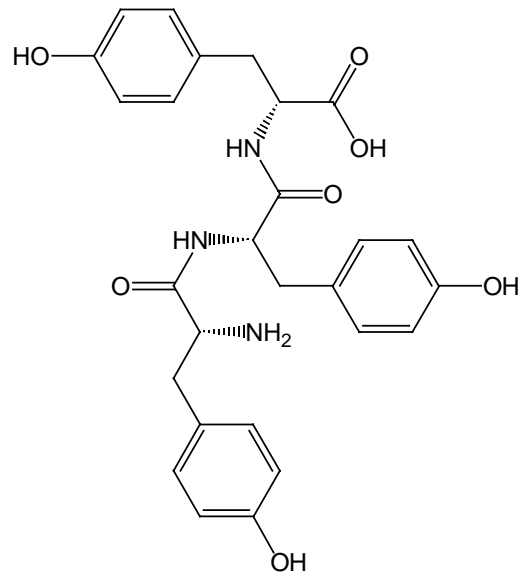

(c)

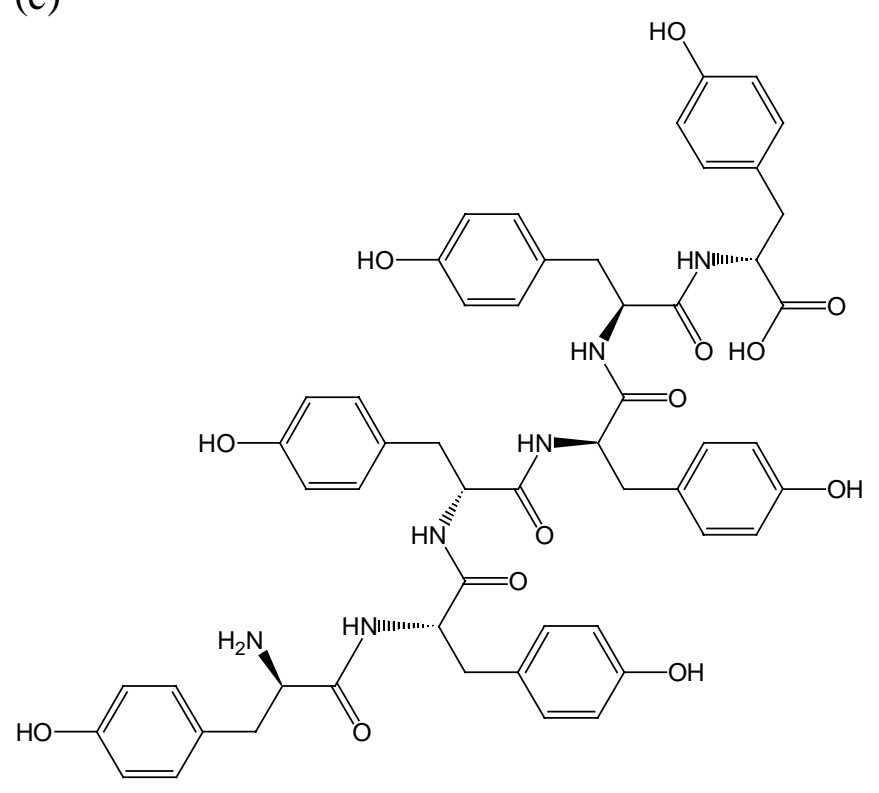


Scheme 3. Structures of (a) Phe-Phe (FF), (b) Phe-Phe-Phe (FFF), (c) Phe-Phe-Phe-Phe (FFFF).

(a)

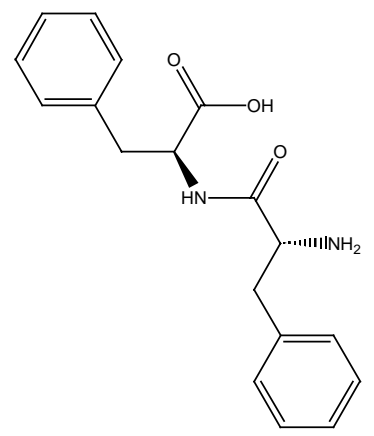

(b)

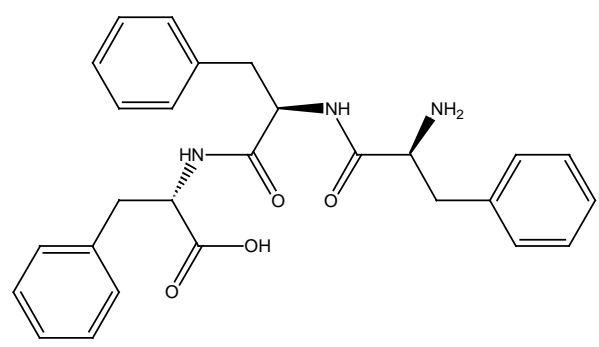

(c)

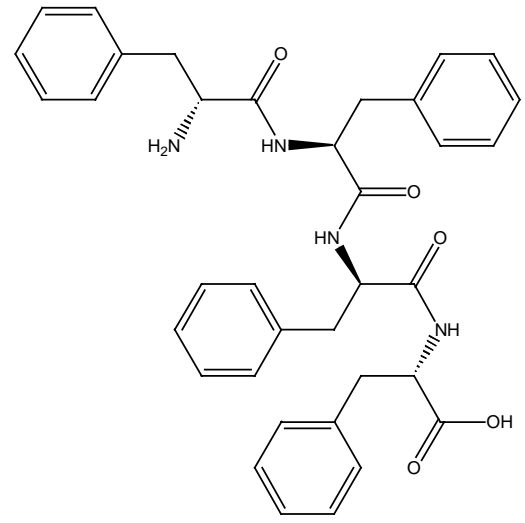


Scheme 4. Structure of (a) Gly-Gly-Gly, (b) Gly-Gly-Gly-Gly (GGGG), (c) Gly-GlyGly-Gly-Gly (GGGGG), (d) Gly-Gly-Gly-Gly-Gly-Gly (GGGGGG)

(a)

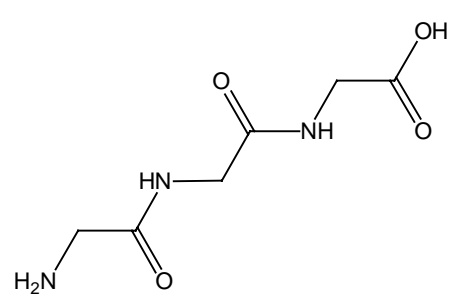

(c)

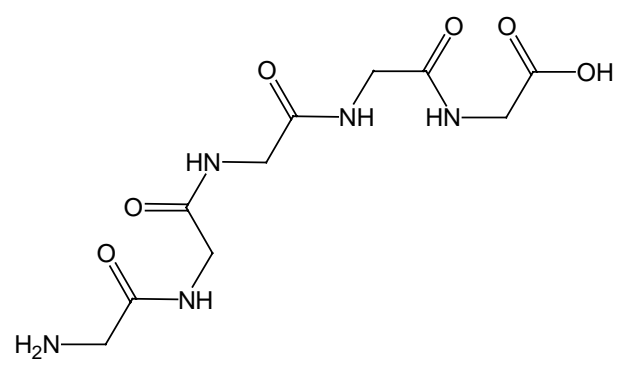

(d)

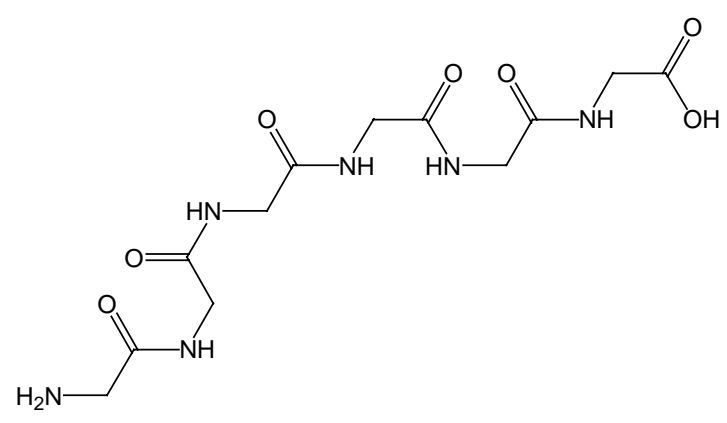

(b)

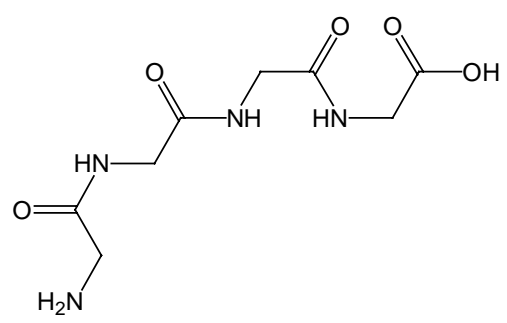


Scheme 5. Structure of Try-Leu (YL)

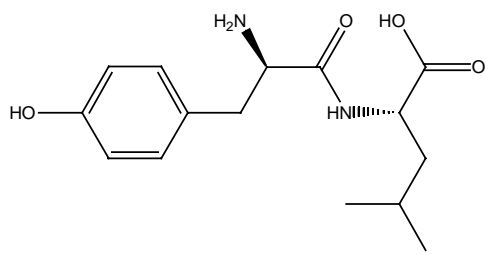




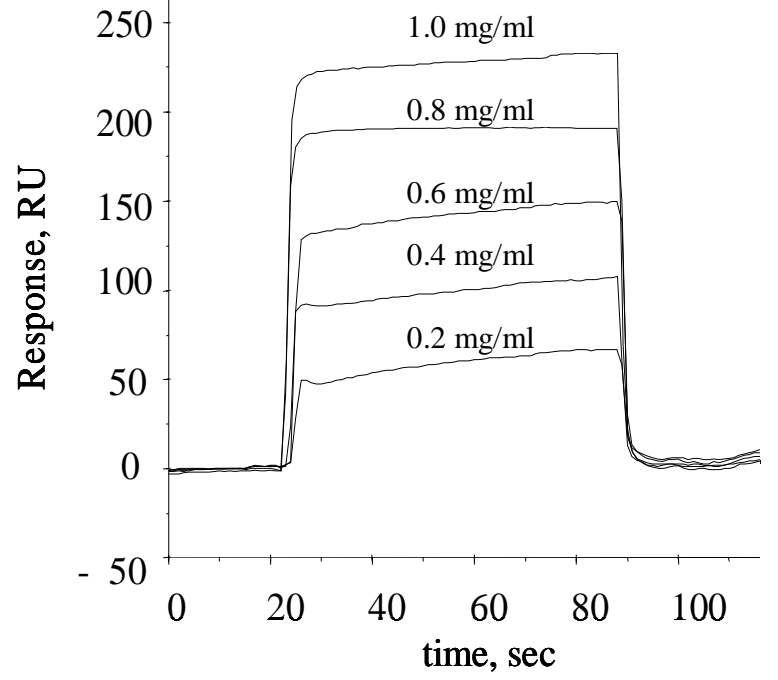

Figure 1. Response curves (sensorgrams) for Tyr-Tyr adsorbing on the PVP surface at $25^{\circ} \mathrm{C}$ from $\mathrm{pH} 7 \mathrm{HEPES}$ buffer solutions. 


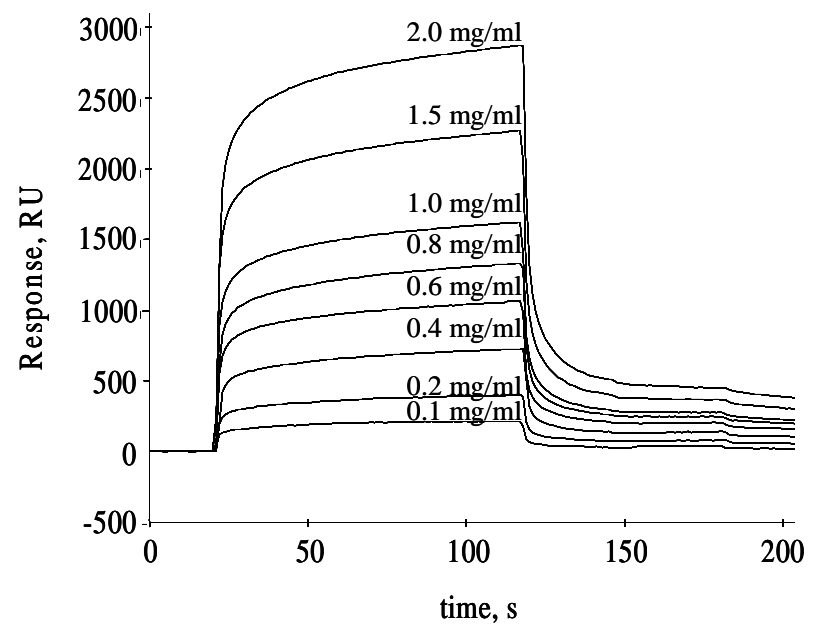

Figure 2. Response curves (sensorgrams) for Tyr-Tyr-Tyr adsorbing on the PVP surface at $25^{\circ} \mathrm{C}$ from $\mathrm{pH} 7 \mathrm{HEPES}$ buffer solutions 


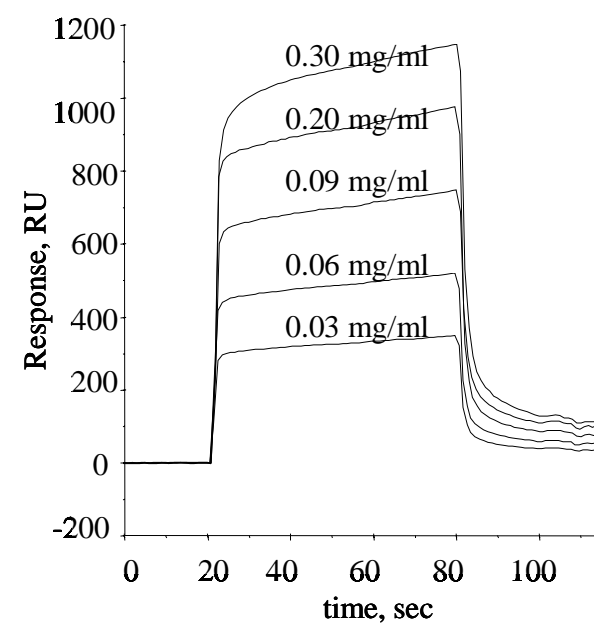

Figure 3. Response curves (sensorgrams) for Tyr-Tyr-Tyr-Tyr-Tyr-Tyr adsorbing on the PVP surface at $25^{\circ} \mathrm{C}$ from $\mathrm{pH} 7$ HEPES buffer solutions. 


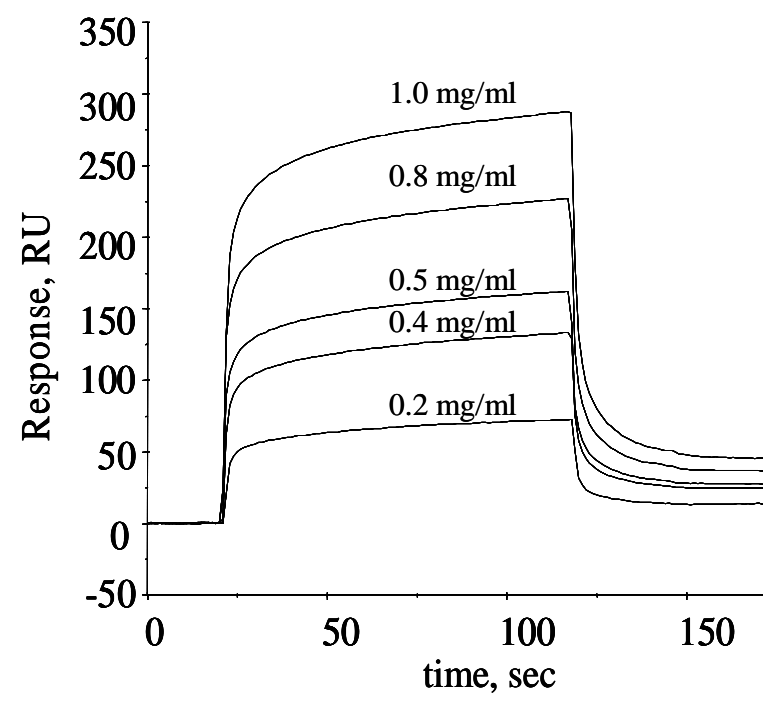

Figure 4. Response curves (sensorgrams) for Phe-Phe adsorbing on the PVP surface at $25^{\circ} \mathrm{C}$ from $\mathrm{pH} 7 \mathrm{HEPES}$ buffer solutions. 


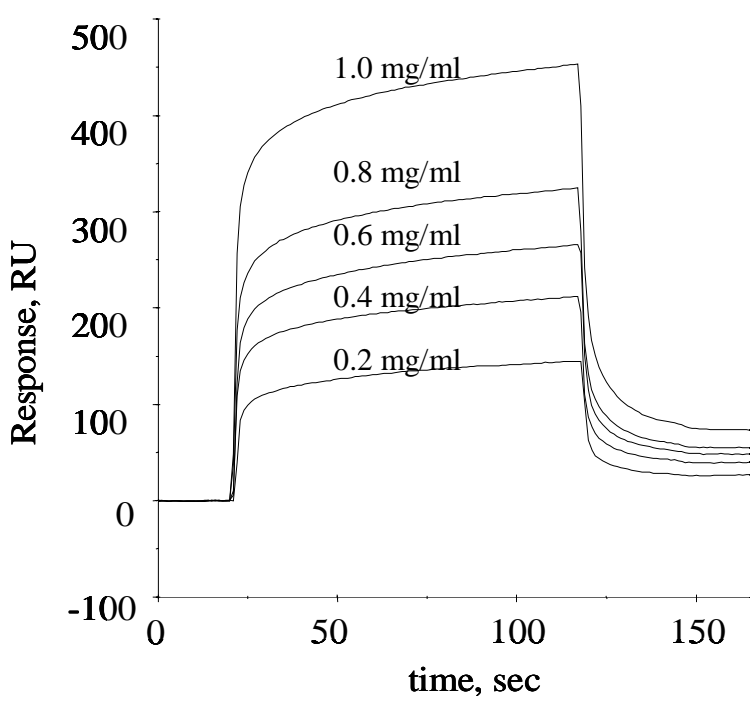

Figure 5. Response curves (sensorgrams) for Phe-Phe-Phe adsorbing on the PVP surface at $25^{\circ} \mathrm{C}$ from $\mathrm{pH} 7$ HEPES buffer solutions. 


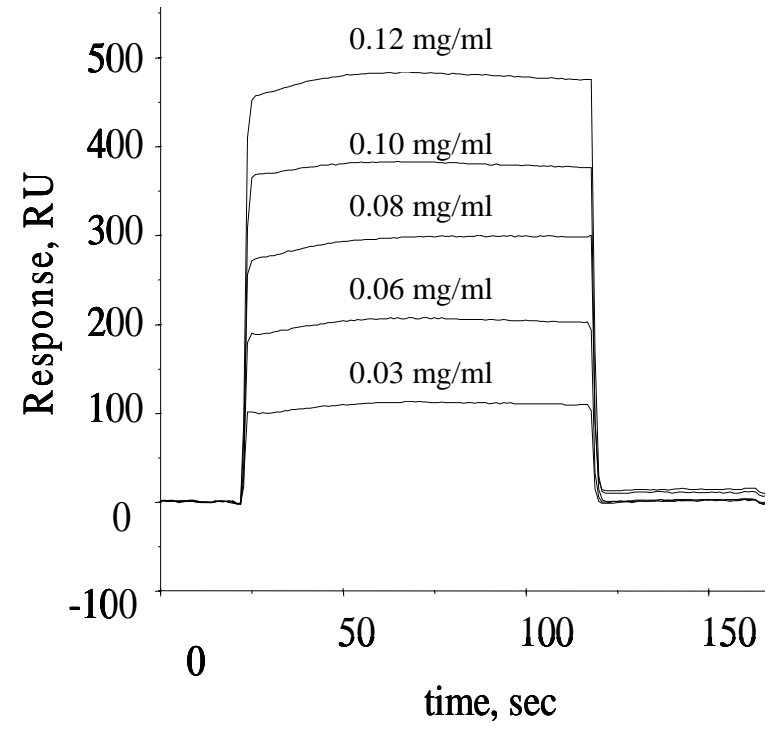

Figure 6. Response curves (sensorgrams) for Phe-Phe-Phe-Phe adsorbing on the PVP surface at $25^{\circ} \mathrm{C}$ from $\mathrm{pH} 7$ HEPES buffer solutions 


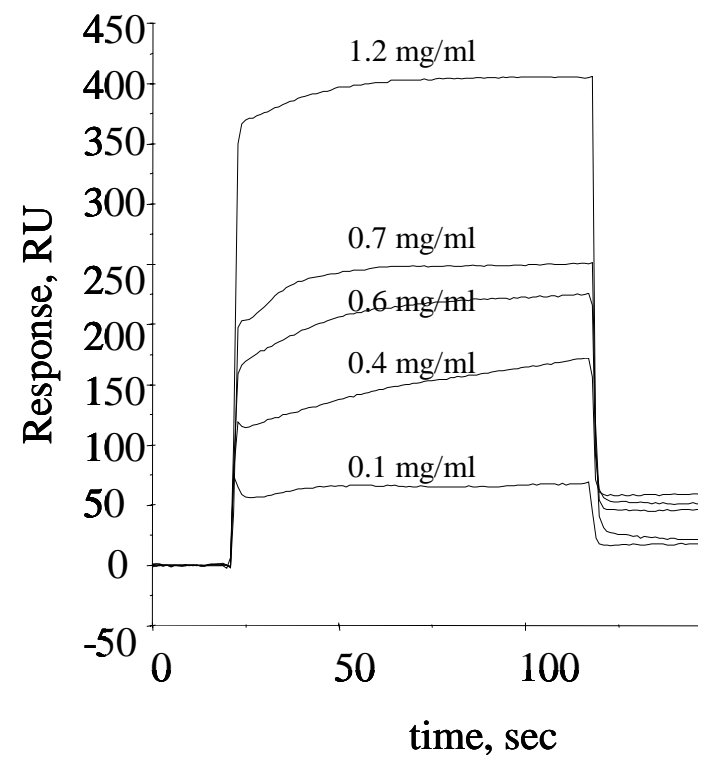

Figure 7. Response curves (sensorgrams) for Gly-Gly-Gly-Gly adsorbing on the PVP surface at $25^{\circ} \mathrm{C}$ from $\mathrm{pH} 7 \mathrm{HEPES}$ buffer solutions. 


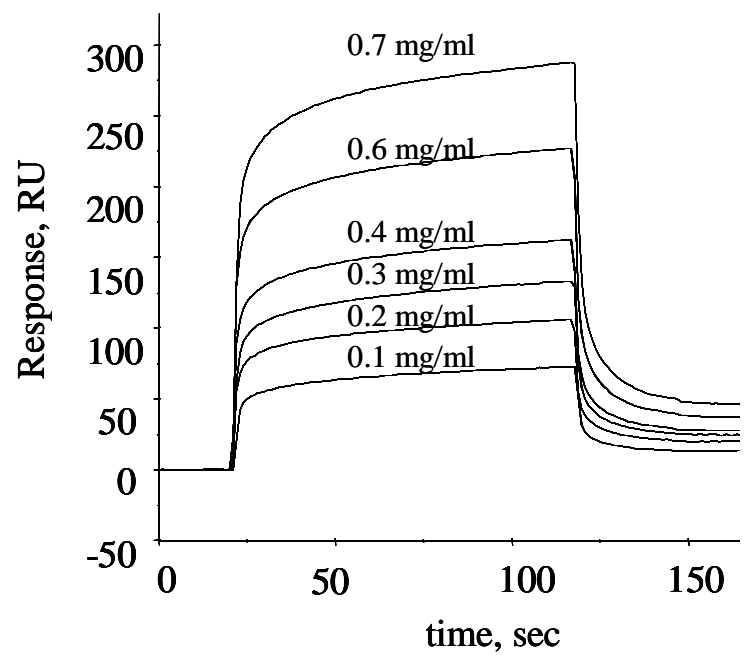

Figure 8. Response curves (sensorgrams) for Gly-Gly-Gly-Gly-Gly adsorbing on the PVP surface at $25^{\circ} \mathrm{C}$ from $\mathrm{pH} 7$ HEPES buffer solutions. 


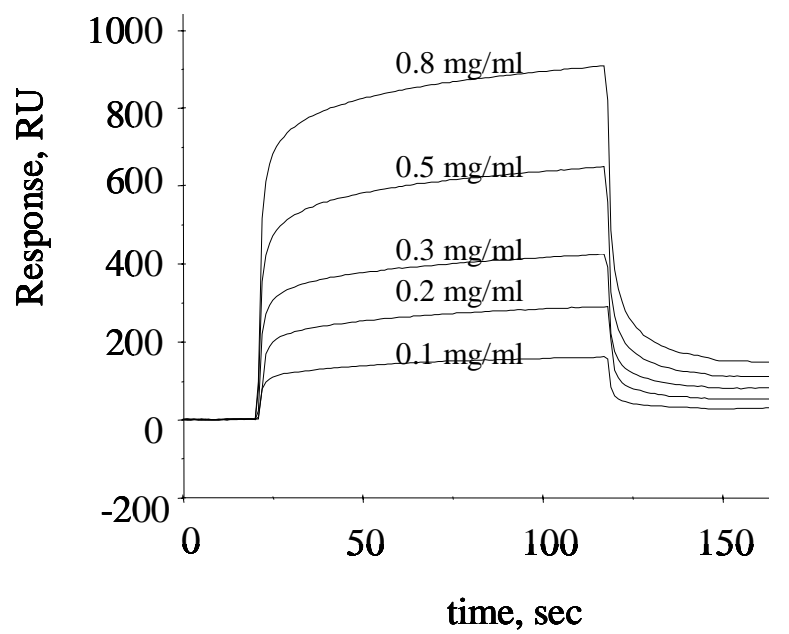

Figure 9. Response curves (sensorgrams) for Gly-Gly-Gly-Gly-Gly-Gly adsorbing on the PVP surface at $25^{\circ} \mathrm{C}$ from $\mathrm{pH} 7$ HEPES buffer solutions. 


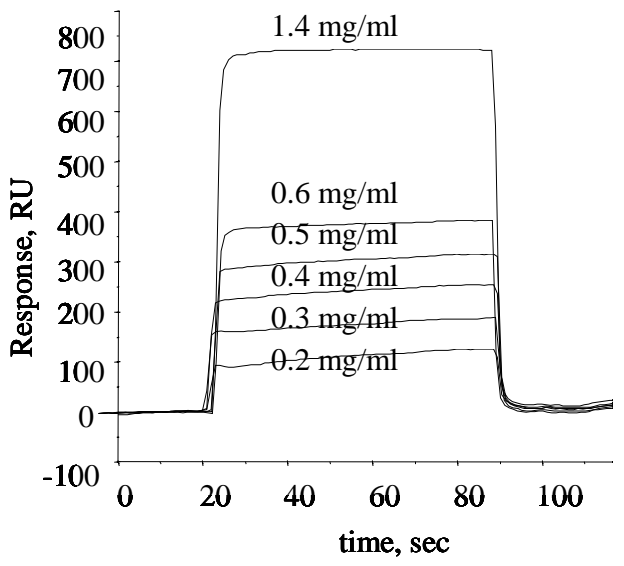

Figure 10. Response curves (sensorgrams) for Tyr-Tyr adsorbing on the PS surface at $25^{\circ} \mathrm{C}$ from $\mathrm{pH} 7 \mathrm{HEPES}$ buffer solution. 


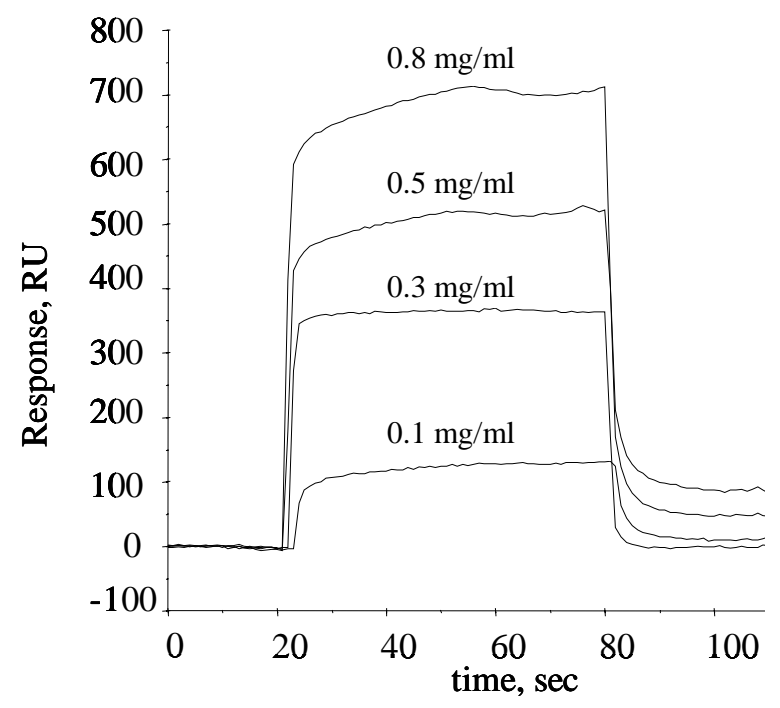

Figure 11. Response curves (sensorgrams) for Tyr-Tyr-Tyr adsorbing on the PS surface at $25^{\circ} \mathrm{C}$ from $\mathrm{pH} 7$ HEPES buffer solutions. 


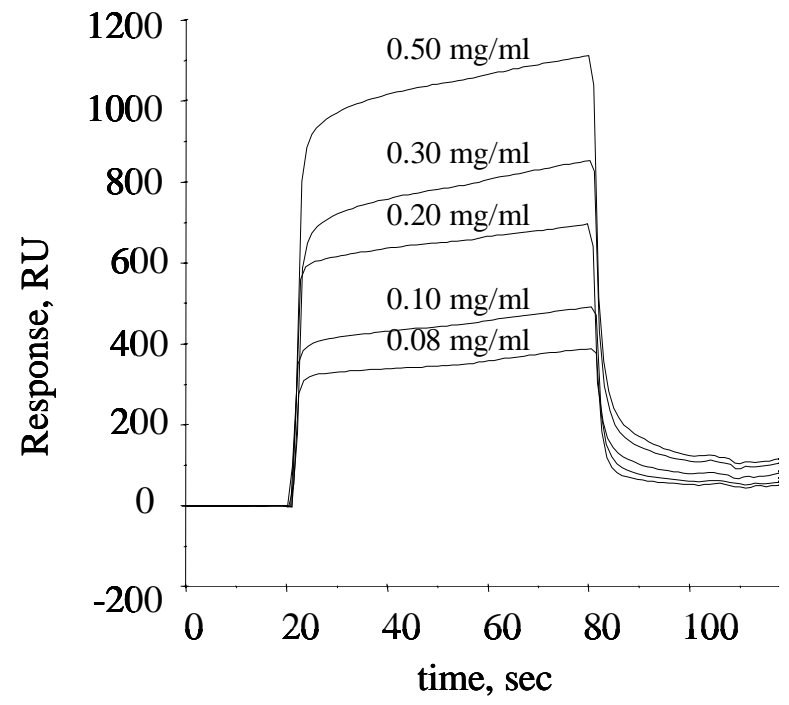

Figure 12. Response curves (sensorgrams) for Tyr-Tyr-Tyr-Tyr-Tyr-Tyr adsorbing on the PS surface at $25^{\circ} \mathrm{C}$ from $\mathrm{pH} 7$ HEPES buffer solutions. 


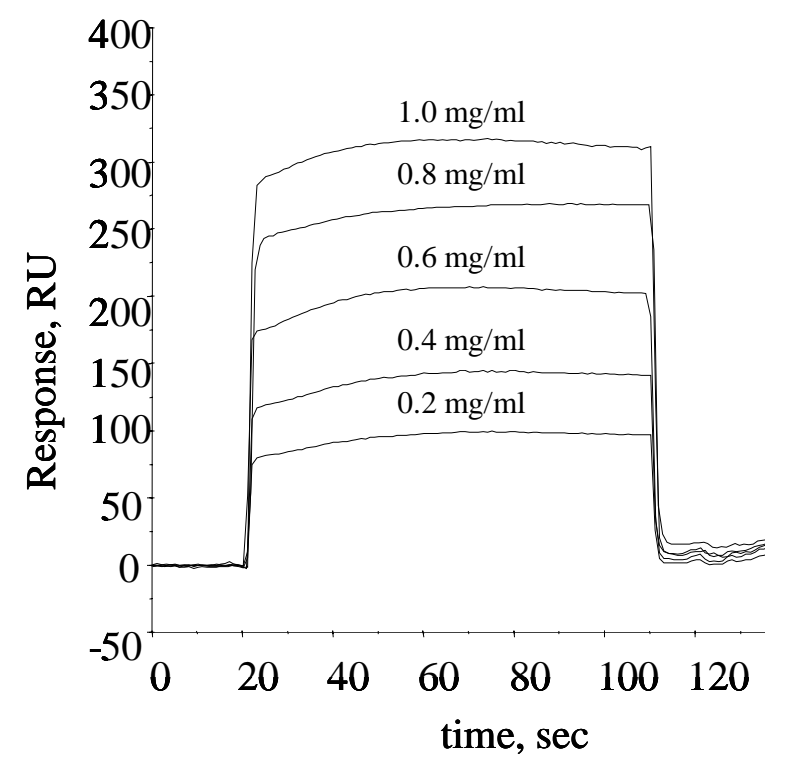

Figure 13. Response curves (sensorgrams) for Phe-Phe adsorbing on the PS surface at $25^{\circ} \mathrm{C}$ from $\mathrm{pH} 7$ HEPES buffer solutions 


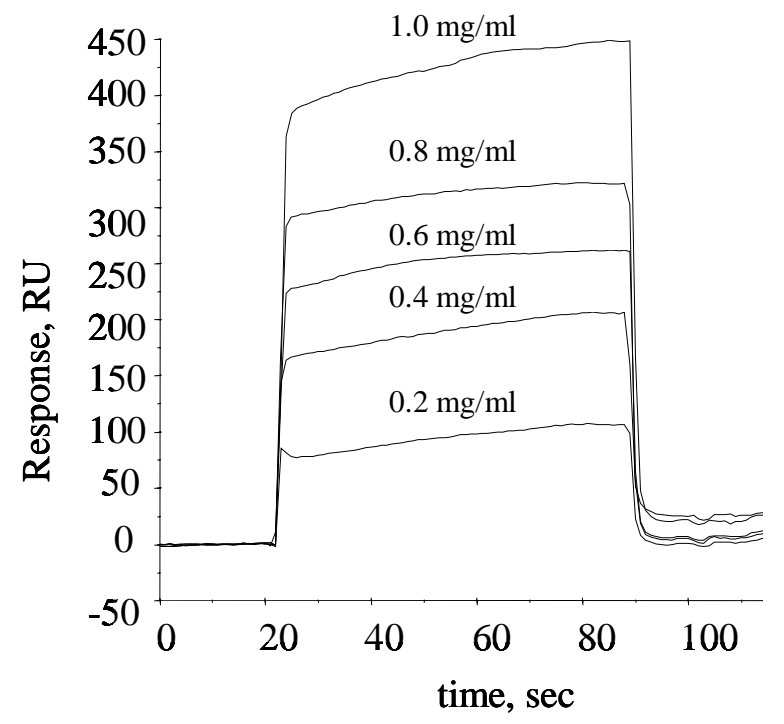

Figure 14. Response curves (sensorgrams) for Phe-Phe-Phe adsorbing on the PS surface at $25^{\circ} \mathrm{C}$ from $\mathrm{pH} 7 \mathrm{HEPES}$ buffer solutions. 


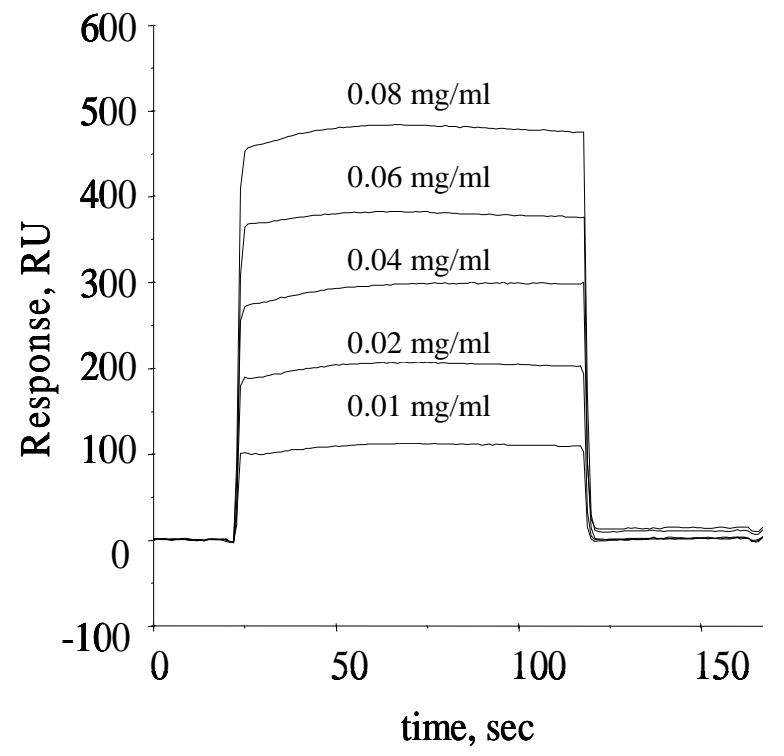

Figure 15. Response curves (sensorgrams) for Phe-Phe-Phe-Phe adsorbing on the PS surface at $25^{\circ} \mathrm{C}$ from $\mathrm{pH} 7 \mathrm{HEPES}$ buffer solutions. 


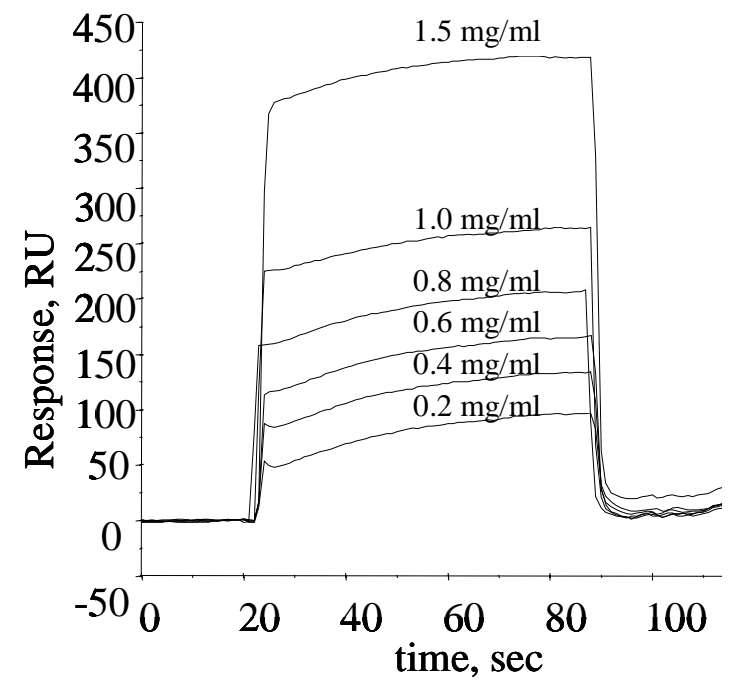

Figure 16. Response curves (sensorgrams) for Gly-Gly-Gly adsorbing on the PS surface at $25^{\circ} \mathrm{C}$ from $\mathrm{pH} 7 \mathrm{HEPES}$ buffer solutions. 


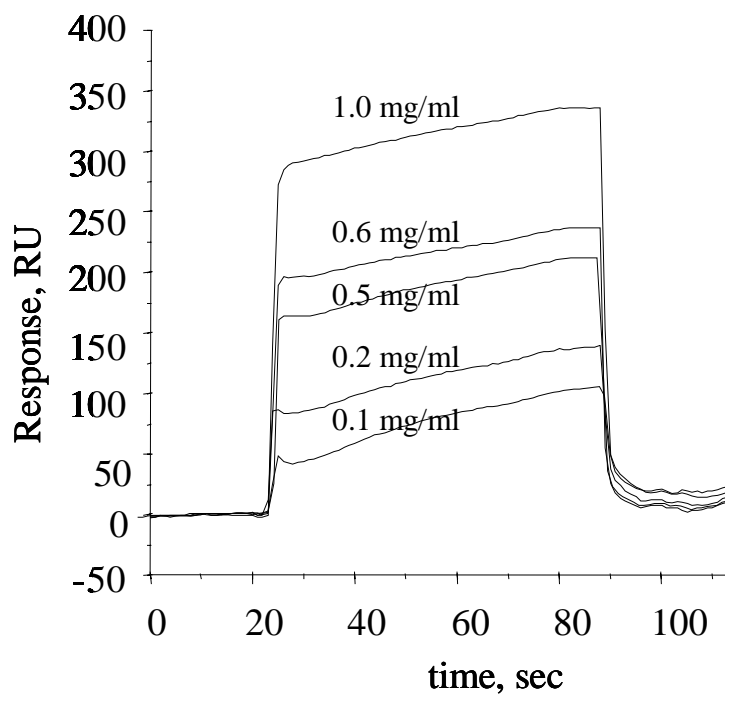

Figure 17. Response curves (sensorgrams) for Gly-Gly-Gly-Gly adsorbing on the PS surface at $25^{\circ} \mathrm{C}$ from $\mathrm{pH} 7$ HEPES buffer solutions. 


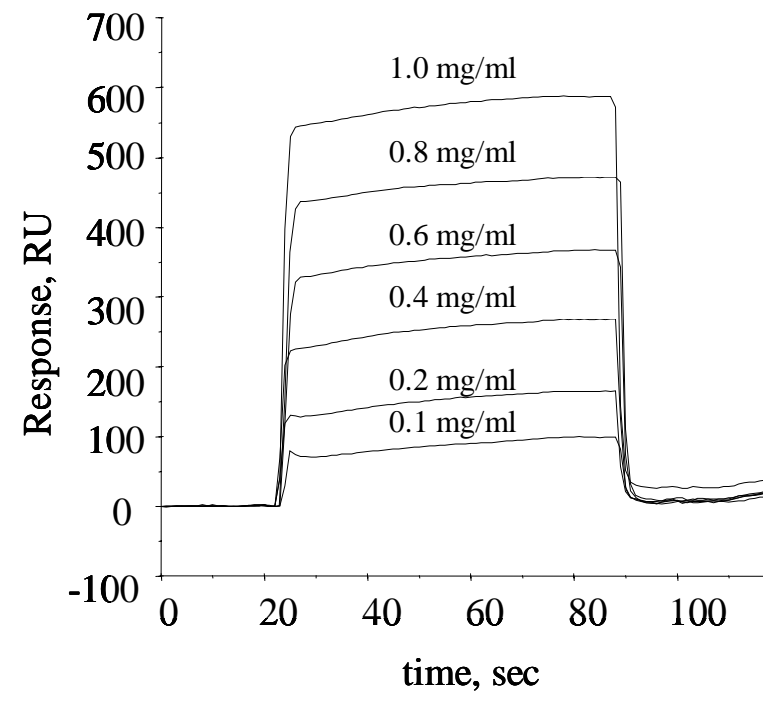

Figure 18. Response curves (sensorgrams) for Gly-Gly-Gly-Gly-Gly adsorbing on the PS surface at $25^{\circ} \mathrm{C}$ from $\mathrm{pH} 7$ HEPES buffer solutions. 


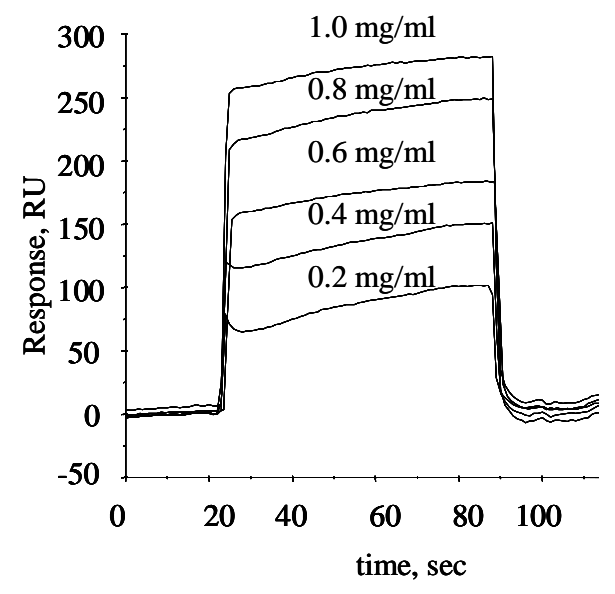

Figure 19. Response curves (sensorgrams) for Tyr-Tyr adsorbing on the BzPVP surface at $25^{\circ} \mathrm{C}$ from $\mathrm{pH} 7 \mathrm{HEPES}$ buffer solutions. 


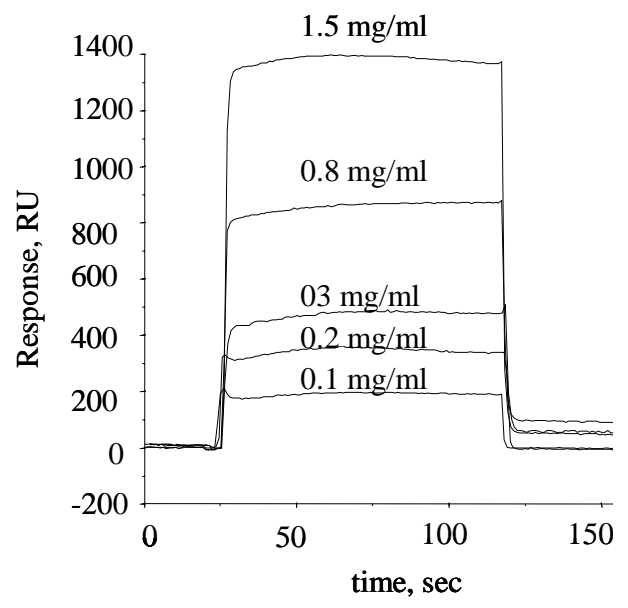

Figure 20. Response curves (sensorgrams) for Tyr-Tyr-Tyr adsorbing on the BzPVP surface at $25^{\circ} \mathrm{C}$ from $\mathrm{pH} 7$ HEPES buffer solutions. 


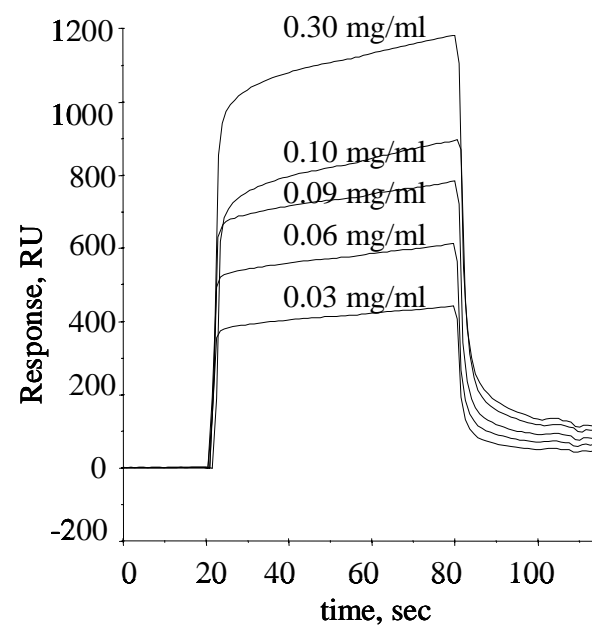

Figure 21. Response curves (sensorgrams) for Tyr-Tyr-Tyr-Tyr-Tyr-Tyr adsorbing on the BzPVP surface at $25^{\circ} \mathrm{C}$ from $\mathrm{pH} 7$ HEPES buffer solutions 


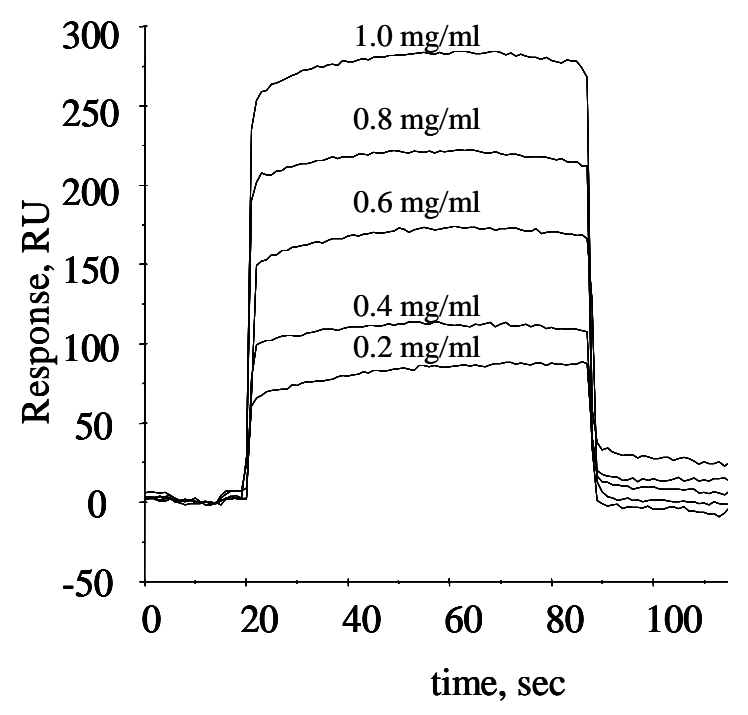

Figure 22. Response curves (sensorgrams) for Phe-Phe adsorbing on the BzPVP surface at $25^{\circ} \mathrm{C}$ from $\mathrm{pH} 7$ HEPES buffer solutions. 


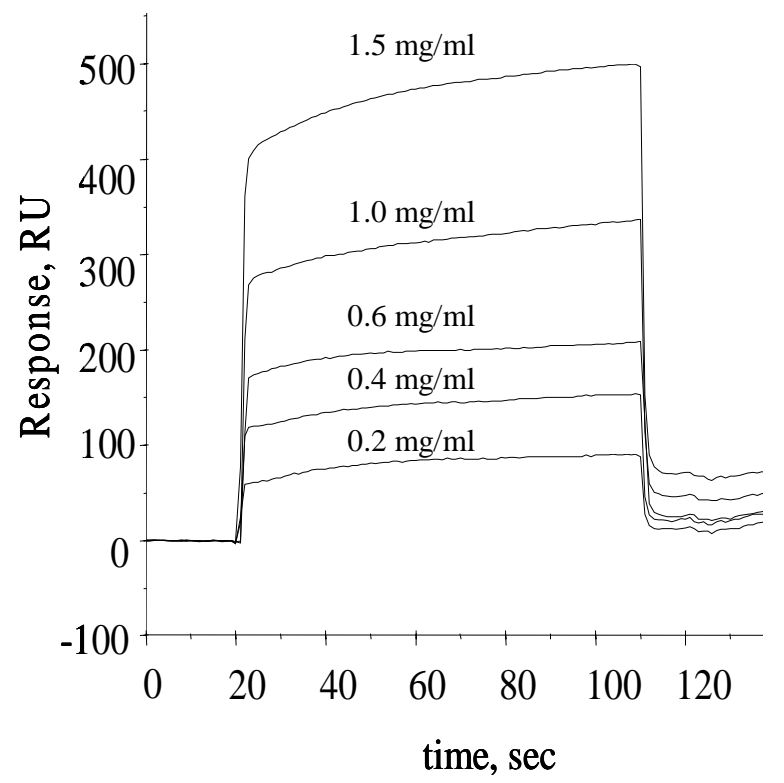

Figure 23. Response curves (sensorgrams) for Phe-Phe-Phe adsorbing on the BzPVP surface at $25^{\circ} \mathrm{C}$ from pH 7 HEPES buffer solution. 


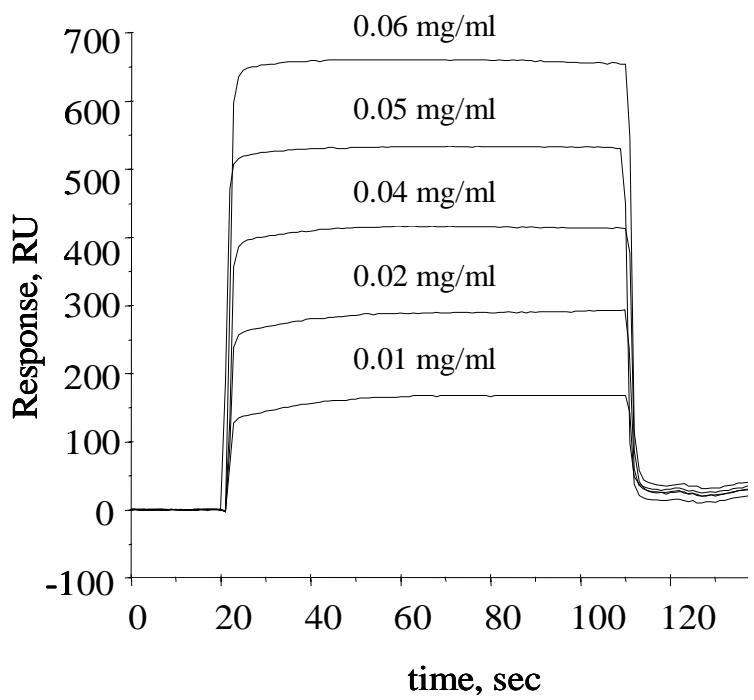

Figure 24. Response curves (sensorgrams) for Phe-Phe-Phe-Phe adsorbing on the BzPVP surface at $25^{\circ} \mathrm{C}$ from $\mathrm{pH} 7$ HEPES buffer solutions. 


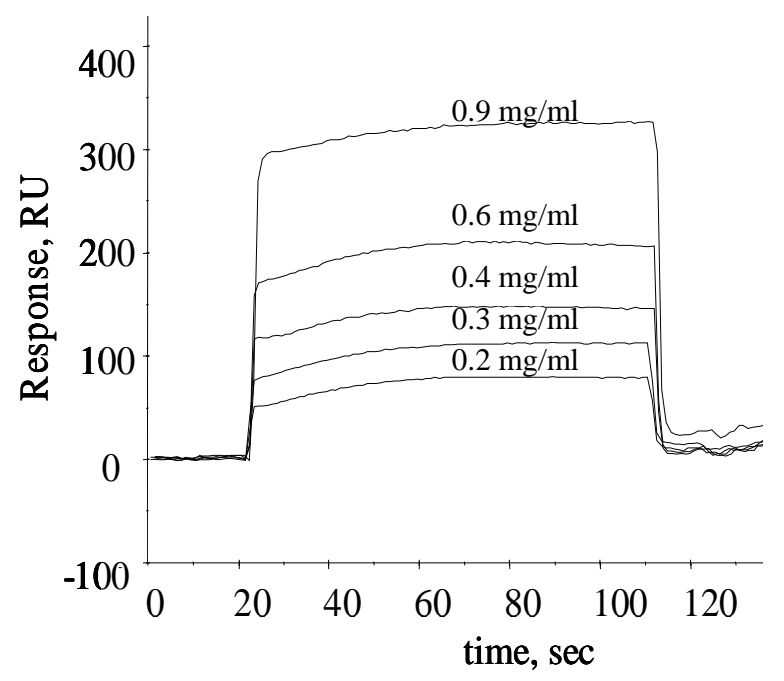

Figure 25. Response curves (sensorgrams) for Gly-Gly-Gly-Gly adsorbing on the BzPVP surface at $25^{\circ} \mathrm{C}$ from $\mathrm{pH} 7$ HEPES buffer solutions. 


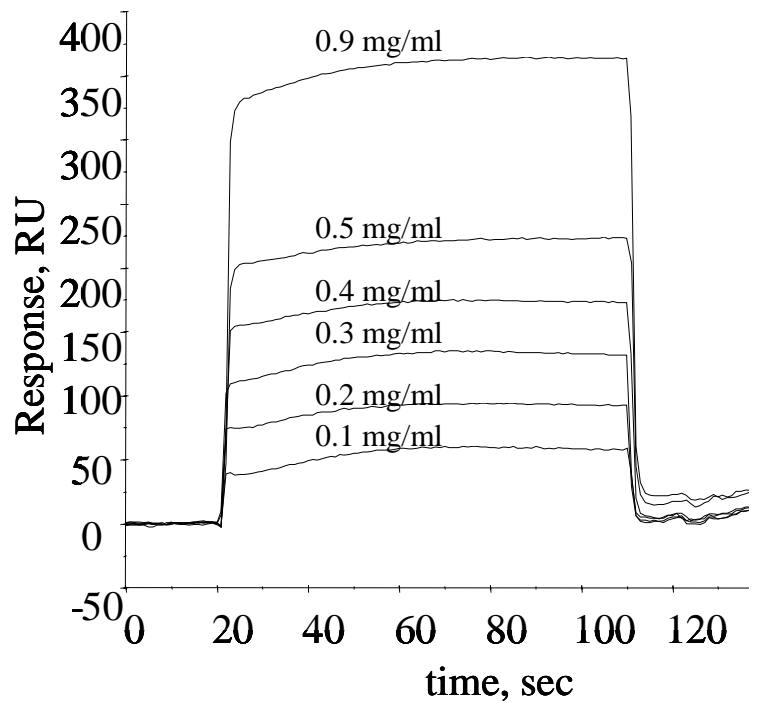

Figure 26. Response curves (sensorgrams) for Gly-Gly-Gly-Gly-Gly adsorbing on the BzPVP surface at $25^{\circ} \mathrm{C}$ from $\mathrm{pH} 7$ HEPES buffer solutions. 


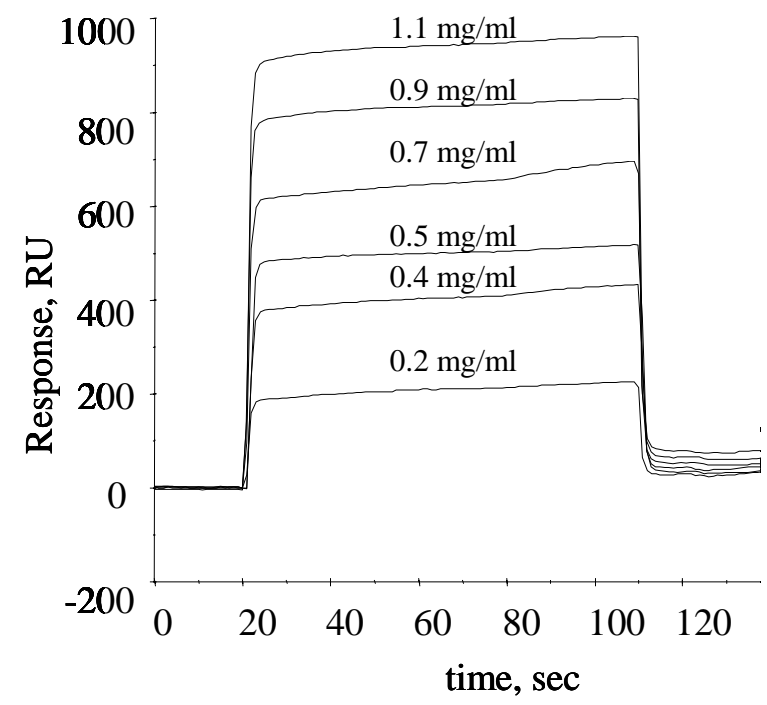

Figure 27. Response curves (sensorgrams) for Gly-Gly-Gly-Gly-Gly-Gly adsorbing on the BzPVP surface at $25^{\circ} \mathrm{C}$ from $\mathrm{pH} 7$ HEPES buffer solutions. 


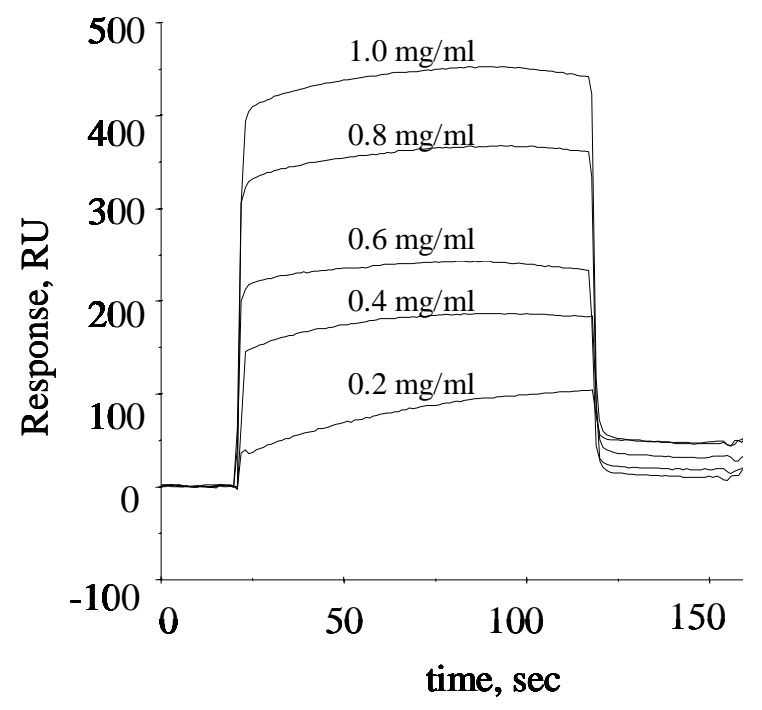

Figure 28. Response curves (sensorgrams) for leucine-tyrosine adsorbing on the PVP surface at $25^{\circ} \mathrm{C}$ from $\mathrm{pH} 7 \mathrm{HEPES}$ buffer solutions. 


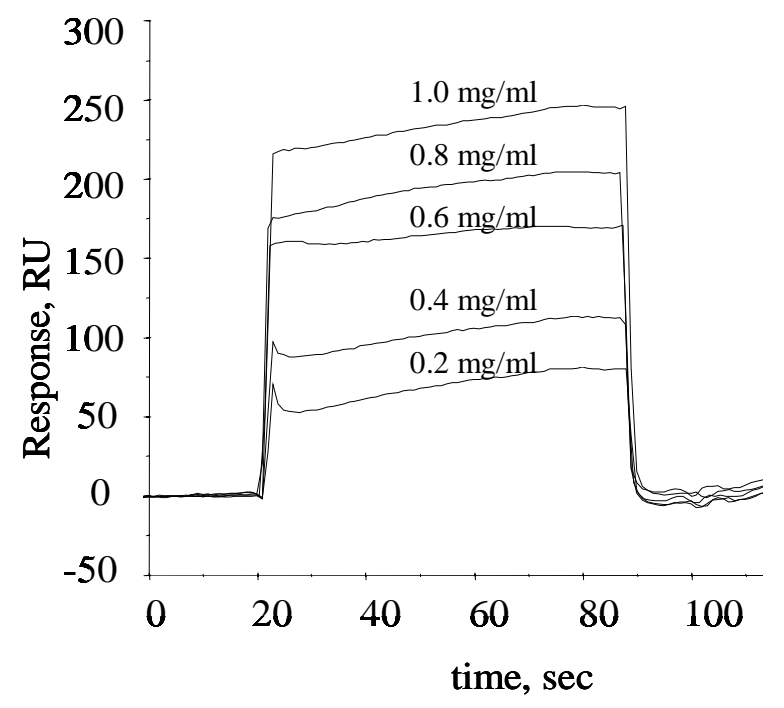

Figure 29. Response curves (sensorgrams) for leucine-tyrosine adsorbing on the PS surface at $25^{\circ} \mathrm{C}$ from $\mathrm{pH} 7$ HEPES buffer solutions 


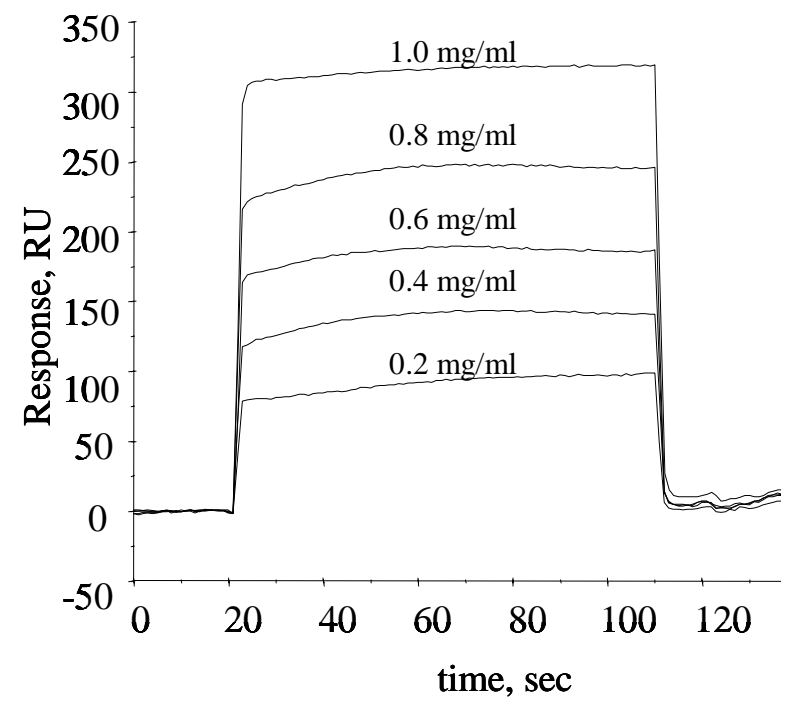

Figure 30. Response curves (sensorgrams) for leucine-tyrosine adsorbing on the BzPVP surface at $25^{\circ} \mathrm{C}$ from $\mathrm{pH} 7 \mathrm{HEPES}$ buffer solutions 


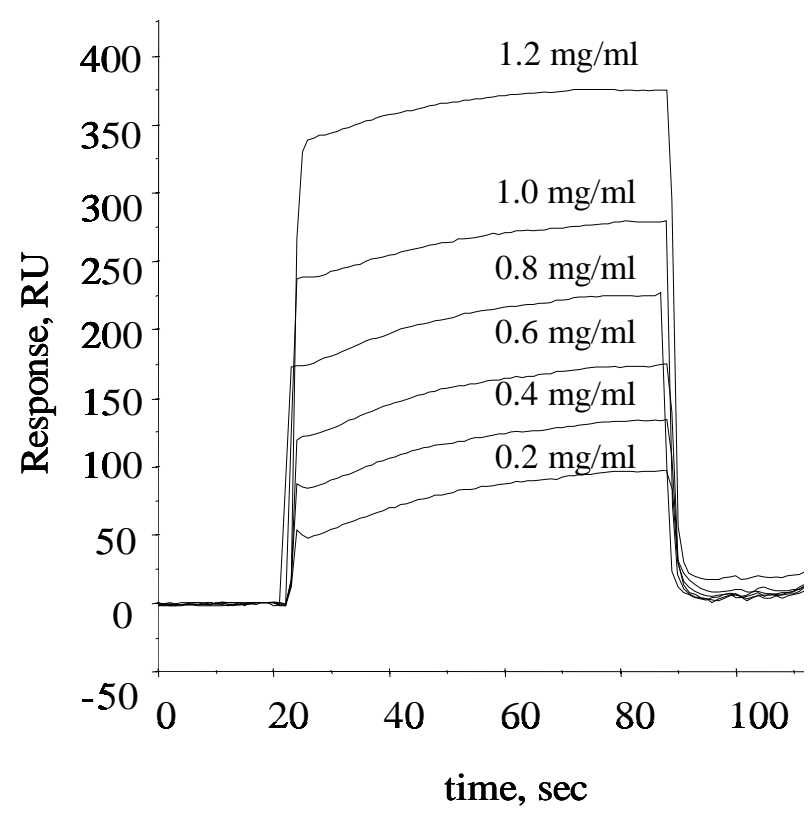

Figure 31. Response curves (sensorgrams) for leucine enkephalin adsorbing on the PVP surface at $25^{\circ} \mathrm{C}$ from $\mathrm{pH} 7 \mathrm{HEPES}$ buffer solutions. 


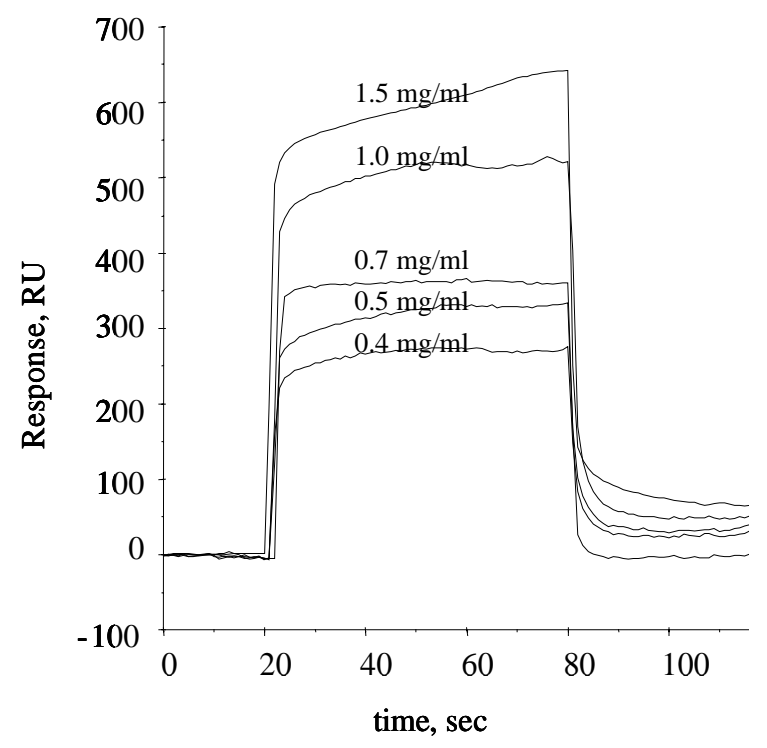

Figure 32. Response curves (sensorgrams) for leucine enkephalin adsorbing on the PS surface at $25^{\circ} \mathrm{C}$ from $\mathrm{pH} 7 \mathrm{HEPES}$ buffer solution 


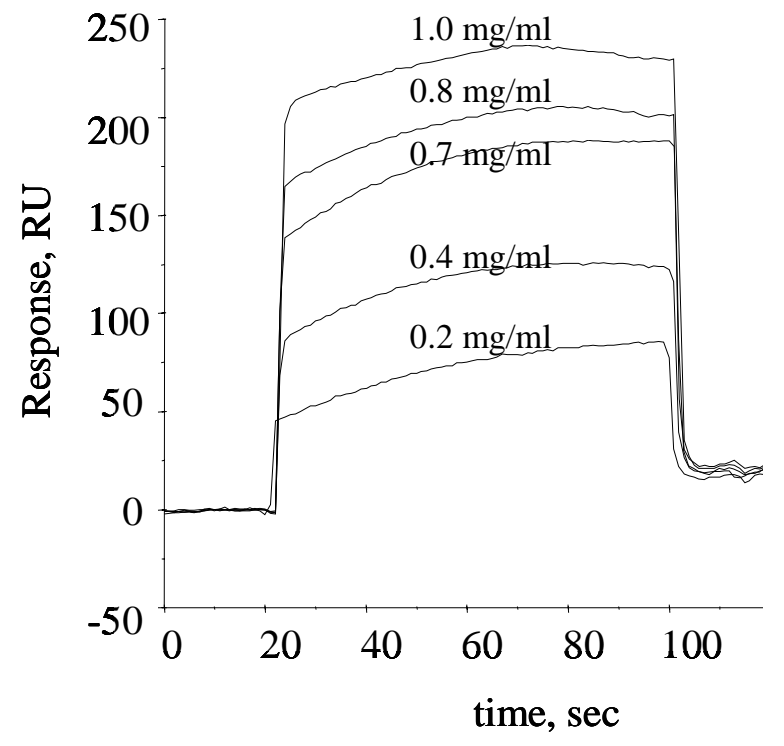

Figure 33. Response curves (sensorgrams) for leucine enkephalin adsorbing on the BzPVP surface at $25^{\circ} \mathrm{C}$ from $\mathrm{pH} 7$ HEPES buffer solutions. 